\title{
Double Interatrial Septum appearing as an Atrial Myxoma: A Case Report and Review of the Literature
}

\author{
1 Julie Wyrobek, ${ }^{2}$ Chales H Brown IV, ${ }^{3}$ Megan P Kostibas, ${ }^{4}$ Susan A Mayer, ${ }^{5}$ Duke E Cameron, ${ }^{6}$ Heather K Hayanga
}

\begin{abstract}
A double interatrial septum (DIS) is a rare finding during echocardiographic evaluation and can often be mistaken for other more common atrial anomalies. The interatrial cavity created by the septum creates a low-flow state that increases risk of thrombus formation and thromboembolic events. Transesophageal echocardiography (TEE) plays a vital role in accurate diagnosis as a DIS is often not seen during transthoracic echocardiography (TTE). In this case, we report a patient who presented for surgery with a preoperative diagnosis of an atrial myxoma, was instead discovered to have a DIS, and then subsequently underwent DIS resection without complication. We discuss the differential of a DIS, including an atrial septal pouch, cor triatriatum, atrial myxoma, and aneurysmal interatrial septum and the classic features of each anomaly for appropriate diagnosis and management.
\end{abstract}

Keywords: Case report, Double interatrial septum, Transesophageal echocardiography.

How to cite this article: Wyrobek J, Brown CH IV, Kostibas MP, Mayer SA, Cameron DE, Hayanga HK. Double Interatrial Septum appearing as an Atrial Myxoma: A Case Report and Review of the Literature. J Perioper Echocardiogr 2017;5(1):16-20.

\section{Source of support: Nil}

Conflict of interest: None

\section{INTRODUCTION}

A DIS is a rare condition with fewer than 20 cases reported in the literature. It is characterized by two

${ }^{1}$ Fellow, ${ }^{2,4,6}$ Assistant Professor, ${ }^{3}$ Instructor, ${ }^{5}$ Senior Professor

${ }^{1}$ Department of Anesthesiology, Perioperative and Pain Medicine Brigham and Women's Hospital, Boston, Massachusetts, USA

${ }^{2,3}$ Department of Anesthesiology and Critical Care Medicine Johns Hopkins Hospital, Baltimore, Maryland, USA

${ }^{4}$ Division of Echocardiology Laboratory, Department of Cardiology The Johns Hopkins Hospital Bayview Medical Center, Baltimore Maryland, USA

${ }^{5}$ Department of Cardiac Surgery, Johns Hopkins Hospital Baltimore, Maryland, USA

${ }^{6}$ Division of Cardiac Anesthesia, Department of Anesthesiology University of Pittsburgh Medical Center, Pittsburgh, Pennsylvania USA

Corresponding Author: Julie Wyrobek, Fellow, Department of Anesthesiology, Perioperative and Pain Medicine, Brigham and Women's Hospital, Boston, Massachusetts, USA, Phone: +6177328210, e-mail: julie.wyrobek@gmail.com parallel interatrial septae forming an echolucent, distinct interatrial cavity between the left and right atrium. ${ }^{1}$ Patients can present with chest pain, transient ischemic attacks, cerebrovascular accidents, and coronary emboli, although several cases have reported a DIS as an incidental finding. These cases can be mistaken for other interatrial anomalies on echocardiography including an atrial septal pouch, cor triatriatum, atrial myxoma, or aneurysmal interatrial septum. The management of these patients has been variable and a standard approach has yet to be established. In this case report, we describe the unexpected TEE finding of a DIS in a patient undergoing surgery for a presumed atrial myxoma in which surgical resection was the management of choice. Careful consideration must be taken during the preoperative echocardiographic assessment of patients with atrial pathology to ensure the correct diagnosis and management strategy.

\section{CASE REPORT}

A 47-year-old female presented to her primary care physician with nonexertional chest tightness and mild fatigue. Her medical history included gastroesophageal reflux disease, dyslipidemia, cervical spine disease, general anxiety, and tobacco abuse. Both of her parents carried diagnoses of atrial fibrillation and hypertension. Chest auscultation revealed a cardiac murmur and she was referred to a local cardiologist. Transthoracic echocardiography demonstrated a broad-based mass measuring $1.0 \times 0.7 \mathrm{~cm}$ with central lucency arising from the interatrial septum in the left atrium (LA) just inferior to the area of the fossa ovalis. The report stated it was suspicious, but not typical, for an atrial myxoma. The patient was referred to our center for a second opinion. A repeat TTE was performed confirming a $1.2 \times 0.9 \mathrm{~cm}$ echogenic mass attached to the midportion of the interatrial septum in the LA, most consistent with an atrial myxoma. No other significant cardiac abnormalities were visualized on TTE. The patient was scheduled for atrial myxoma resection.

The patient underwent an uneventful anesthetic induction and endotracheal intubation, and arterial and central lines were placed. Transesophageal echocardiography revealed a membranous, mobile projection on the interatrial septum in the LA measuring $1.0 \times 2.0 \mathrm{~cm}$ (Fig. 1) with 
flow communication between the DIS cavity and the LA. There was no patent foramen ovale (PFO) present, or any other communication with the right atrium. No clot was visualized within the DIS or LA. There was trace mitral regurgitation present and no other valvular pathology noted. The cardiac anesthesiology team, with further consultation by cardiology and cardiothoracic surgery teams, raised concern that the findings were more consistent with a DIS than with an atrial myxoma. The family and health care proxy was updated and, after deliberation, the decision was made to proceed with surgical excision. This was in part due to the uncertain diagnosis, but also because without resection, lingering concerns about thrombus formation and embolization would remain. Total cardiac bypass time was 62 minutes and total aortic cross clamp time was 36 minutes. The surgical team was able to visualize and confirm a redundant, duplicated atrial septum. The pathology report stated the tissue was an atrial septum excision with benign endocardium and myocardium negative for tumor. The trachea was extubated on day 1 of surgery. The patient was discharged home on postoperative day 5 on aspirin and a beta-blocker. Follow-up at 1 month was unremarkable with resolution of initial symptoms.
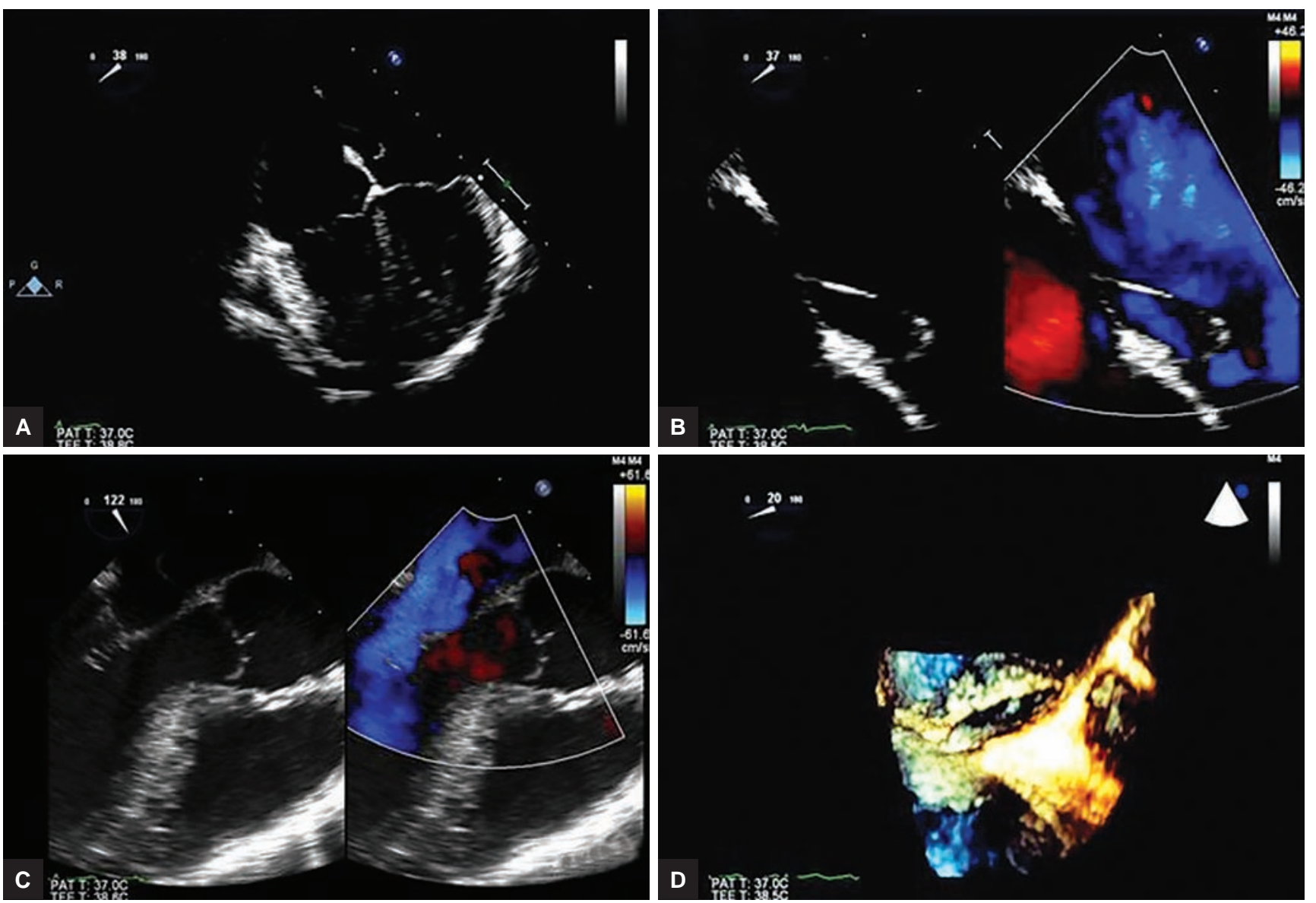

Figs 1A to D: (A) Midesophageal four-chamber view of DIS; (B) magnified view of DIS with color flow Doppler; (C) aortic valve long-axis view; and (D) three-dimensional view of DIS visualized using transesophageal echo 
of the interatrial cavity with the LA compared with the right is usually noted. ${ }^{3}$ Identification is best seen in the TTE subcostal view or the TEE midesophageal bicaval view. In most cases, TEE evaluation was necessary for diagnostic confirmation.

A DIS may be an isolated finding, but may also be accompanied by abnormalities including a PFO, mitral atresia, a ventricular septal defect, inferior vena cava stenosis, or hypoplastic left heart. ${ }^{1,4}$ Given its proclivity to a low-flow state, thrombus formation leading to embolic stroke is of great concern and thrombus formation in the DIS has been reported. ${ }^{5}$ Cases of transient ischemic attack, cerebrovascular accident, and a coronary embolus from presumed emboli have also been attributed to the low-flow state created by a DIS. ${ }^{4,5}$

Differential diagnosis for a DIS includes a left atrial septal pouch, cor triatriatum, an atrial myxoma, or an aneurysmal interatrial septum. Several distinct anatomical differences exist between these conditions. A left atrial septal pouch (Fig. 2A) is a very common, yet newly described anomaly. ${ }^{6,7}$ Shortly after birth, fusion of the septum secundum and septum primum occurs with closure of the foramen ovale. The merging is thought to occur in a caudal to cranial direction. ${ }^{6}$ When these layers only fuse at the caudal zone, a blind recess or pouch in the LA is formed cranially between the septum secundum and the flap-like unfused septum primum. In contrast to a DIS, this anomaly is not typically associated with a PFO, though it has been described in the literature. ${ }^{8,9}$ While color flow mapping is often easily seen within a DIS given its large structure, it can be very difficult to obtain with a left atrial septal pouch. ${ }^{8}$ Three-dimensional TEE has greater sensitivity in detecting a left atrial septal pouch in patients in comparison with two-dimensional TEE. ${ }^{10}$ Similar to a DIS, blood flow stasis and thrombus formation are of great concern. Thrombus formation within the pouch is considered a potential source of thromboembolism, though studies have shown inconclusive results as to whether a left atrial septal pouch increases risk of cerebrovascular events. ${ }^{7}$ A left atrial septal pouch thrombus has also previously been mistaken as a myxoma on TEE. ${ }^{11}$ Similar to a DIS, management of these patients has yet to be standardized, but anticoagulation and surgical interventions have been described. ${ }^{8}$

Cor triatriatum sinistrum (Fig. 2B) is a division in the LA impeding direct communication between the pulmonary veins and the LA with creation of a posteriorsuperior and anterior-inferior chamber within the LA. ${ }^{12}$ These chambers communicate through perforations in the fibromuscular membrane. This condition is thought to arise from abnormal incorporation of the common pulmonary vein. ${ }^{13}$ In contrast to a DIS, the additional tissue is not parallel to the interatrial septum. ${ }^{3}$ Associated echocardiographic findings include atrial septal defect, PFO, left atrial enlargement, and mitral regurgitation. ${ }^{14}$ This condition is commonly discovered in infants. Adult asymptomatic cases have infrequently been reported. ${ }^{12,14}$ Cor triatriatum in the adult population has been attributed as a source of thromboembolism including cerebral infarctions and arterial occlusions. ${ }^{14}$ Surgical repair is the treatment of choice for patients presenting with thromboembolic events, although asymptomatic patients have been managed medically on anticoagulation. ${ }^{12,14}$

An atrial myxoma (Fig. 2C) is a benign intracavitary, round or ovoid tumor that occurs in approximately $0.03 \%$ of the population..$^{15}$ Patients can present with clinical signs of mitral valve obstruction, constitutional symptoms, such as weight loss, fatigue, or weakness, dyspnea secondary to pulmonary edema, syncope secondary to outflow obstruction, or palpitations or cerebrovascular symptoms secondary to thromboemboli. ${ }^{16,17}$ Several paraneoplastic symptoms have also been associated with cardiac myxomas. ${ }^{17}$ The etiology is unknown, but it is hypothesized that this tumor develops from multipotent mesenchymal cells from both the endocardium and epicardium. ${ }^{15}$ Most commonly, myxomas occur in the LA on the interatrial septum in the region of the fossa ovalis. Size can be variable, with reports describing myxomas as large as $15 \mathrm{~cm}$. Other associated cardiac findings include left atrial hypertrophy, rhythm disorders, and conduction disorders. ${ }^{16}$ Surgical intervention is the treatment modality of choice given its high survival rates between 80 and $98 \%$ at 10 years and lack of studies with anticoagulation as exclusive management. ${ }^{17}$

An aneurysmal interatrial septum (Fig. 2D) ${ }^{7}$ is defined by a thin, very mobile compliant septum at the level of the fossa ovalis that bows between the atria. ${ }^{18}$ Although the etiology is unclear, aneurysmal interatrial septum is thought to be secondary to septal malformation at the region of the fossa ovalis. ${ }^{19}$ Past studies have used an excursion value of greater than $1.0 \mathrm{~cm}$ for diagnosis. ${ }^{18}$ Fenestrations may be visualized in the aneurysm via TEE. ${ }^{20}$ An aneurysmal interatrial septum is thought to occur in 1 to $2.2 \%$ of the population and is often associated with a PFO. ${ }^{18}$ Although an aneurysmal interatrial septum is often found in isolation, other less commonly associated cardiac abnormalities include an atrial septal defect or mitral valve prolapse. ${ }^{19}$ In comparison with a DIS, no interseptal cavity exists. ${ }^{3}$ Aneurysmal interatrial septum can be a source of intracardiac thrombus, as well as an arrhythmogenic focus. ${ }^{20}$ Patients with an aneurysmal interatrial septum have an increased risk for cerebrovascular events. Management for symptomatic patients may 

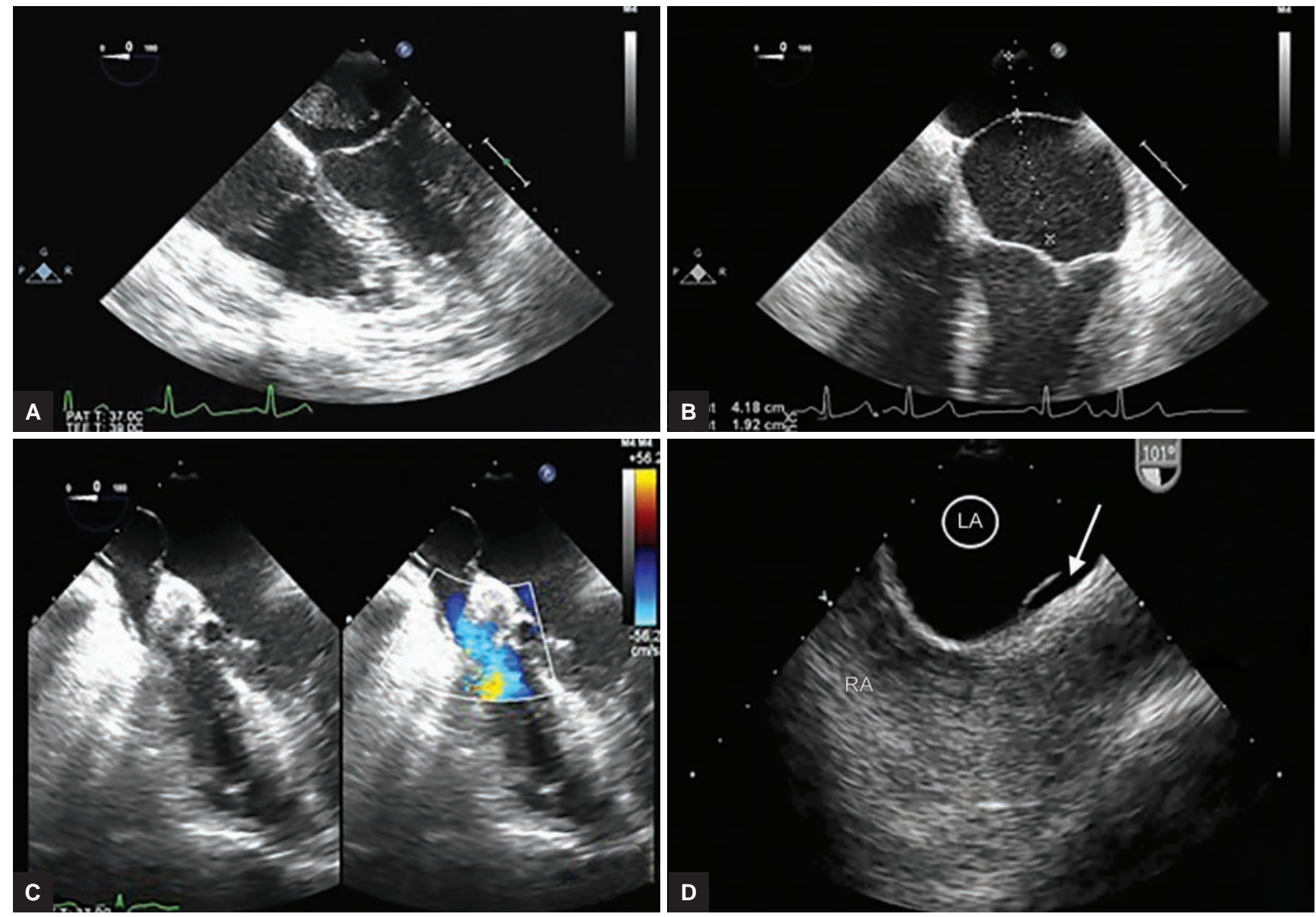

Figs 2A to D: Examples of (A) Atrial myxoma; (B) cor triatriatum sinistrum; (C) aneurysmal interatrial septum; and (D) left atrial septal pouch

include surgical correction or anticoagulation; however, given the prevalence of aneurysmal interatrial septum, this isolated finding on echocardiographic evaluation is often considered incidental and it is unclear at this time whether further workup or management is warranted. ${ }^{18}$ While the atrial abnormalities described above may look similar on imaging, and must be included on the differential diagnosis, we have described the distinctive attributes of each that can be used to guide diagnostic, and thus therapeutic, efforts.

\section{CONCLUSION}

This case of a DIS initially thought to be an atrial myxoma serves to lend insight into the challenges inherent in appropriate diagnosis of a DIS. It describes the role of TEE in attempting to define the appropriate diagnosis among several other conditions with varying risk factors and management strategies. Lastly, it provides another case description of a DIS and an alternative strategy to possible anticoagulation in an attempt to further expand our knowledge of this anatomical finding to best determine future management.

\section{REFERENCES}

1. Roberson DA, Javois AJ, Cui W, Madronero LF, Cuneo BF, Muangmingsuk S. Double atrial septum with persistent interatrial space: echocardiographic features of a rare atrial septal malformation. J Am Soc Echocardiogr 2006 Sep;19(9):1175-1181.

2. Bandyopadhyay S, Sathia S, Sanyal MK, Mandana K. Incidental finding of a double interatrial septum in an elderly female undergoing coronary artery bypass graft surgery. Anesth Analg 2014 Dec;119(6):1267-1270.

3. Kumar PA, Martinelli SM, Kyle RW, Arora H. Echocardiographic discovery of doubled interatrial septum: an incidental finding. Semin Cardiothorac Vasc Anesth 2016 Sep;20(3): 237-239.

4. Li X, Zhu D, Tang H, Feng Y, An Q. Double atrial septum with interatrial chamber formation and recurrent paradoxical embolism. Ann Thorac Cardiovasc Surg 2014 Nov;20 (Suppl):829-831.

5. Breithardt OA, Papavassiliu T, Borggrefe M. A coronary embolus originating from the interatrial septum. Eur Heart J 2006 Dec;27(23):2745.

6. Krishnan SC, Salazar M. Septal pouch in the left atrium: a new anatomical entity with potential for embolic complications. JACC Cardiovasc Interv 2010 Jan;3(1):98-104.

7. Wong JM, Lombardo DM, Barseghian A, Dhoot J, Hundal HS, Salcedo J, Paganini-Hill A, Wong ND, Fisher M . Left atrial 
septal pouch in cryptogenic stroke. Front Neurol 2015 Mar;6:57.

8. Bandyopadhyay S, Mandana K. Left atrial septal pouch: a potential source of systemic thromboembolism: incidental transesophageal echocardiogram findings. Anesth Analg 2015 Jul;121(1):59-61.

9. Kijima Y, Bokhoor P, Tobis JM. Patent foramen ovale with right atrial septal pouch. Catheter Cardiovasc Interv 2017 Apr;89(5):E169-E171.

10. Elsayed M, Thind M, Nanda NC. Two- and three-dimensional transthoracic echocardiographic assessment of tricuspid valve prolapse with mid-to-late systolic tricuspid regurgitation. Echocardiography 2015 Jun;32(6):1022-1025.

11. Shimamoto K, Kawagoe T, Dai K, Inoue I. Thrombus in the left atrial septal pouch mimicking myxoma. J Clin Ultrasound 2014 Mar-Apr;42(3):185-188.

12. Bharucha T, Spicer DE, Mohun TJ, Black D, Henry GW, Anderson RH. Cor triatriatum or divided atriums: which approach provides the better understanding? Cardiol Young 2015 Feb;25(2):193-207.

13. Nassar PN, Hamdan RH. Cor triatriatum sinistrum: classification and imaging modalities. Eur J Cardiovasc Med 2011 Jan;1(3):84-87.

14. Park KJ, Park IK, Sir JJ, Kim HT, Park YI, Tsung PC, Chung JM, Park KI, Cho WH, Choi SK . Adult cor triatriatum presenting as cardioembolic stroke. Intern Med 2009 Jul;48(13): 1149-1152.

15. Di Vito A, Mignogna C, Donato G. The mysterious pathways of cardiac myxomas: a review of histogenesis, pathogenesis and pathology. Histopathology 2015 Feb;66(3):321-332.

16. Pinede L, Duhaut P, Loire R. Clinical presentation of left atrial cardiac myxoma. A series of 112 consecutive cases. Medicine (Baltimore) 2001 May;80(3):159-172.

17. Smith M, Chaudhry MA, Lozano P, Humphrey MB. Cardiac myxoma induced paraneoplastic syndromes: a review of the literature. Eur J Intern Med 2012 Dec;23(8):669-673.

18. Burch TM, Davidson MF, Pereira SJ. Use of transesophageal echocardiography in the evaluation and surgical treatment of a patient with an aneurysmal interatrial septum and an intracardiac thrombus traversing a patent foramen ovale. Anesth Analg 2008 Mar;106(3):769-770.

19. Mügge A, Daniel WG, Angermann C, Spes C, Khandheria BK, Kronzon I, Freedberg RS, Keren A, Denning K, Engberding R, et al. Atrial septal aneurysm in adult patients. A multicenter study using transthoracic and transesophageal echocardiography. Circulation 1995 Jun;91(11):2785-2792.

20. Schneider B, Hanrath P, Vogel P, Meinertz T. Improved morphologic characterization of atrial septal aneurysm by transesophageal echocardiography: relation to cerebrovascular events. J Am Coll Cardiol 1990 Oct;16(4):1000-1009. 